\section{An analysis of individual differences in similarity judgments about complex random forms}

\author{
LARRY G. RICHARDS* \\ University of Virginia, Charlottesville, Virginia 22901
}

A variation of the method of graded dichotomies was used to obtain judgments of interstimulus similarity for all pairings of 21 12-turn random shapes. Using the Tucker-Messick procedure, a six-dimensional spatial model, representing individual variation in performance on the task, was derived. Six "idealized individuals" (IIs) were isolated. A five-dimensional Euclidean spatial model was obtained for each II using the Shepard-Kruskal scaling technique. The resulting psychological dimensions of perceived form were related to two sets of physical measures on the forms: the factor space suggested by Brown and Owen and a set of more basic physical measures. These basic measures allowed interpretation of most of the psychological dimensions and revealed differences between IIs in patterns of cue utilization.

One of the dominant features of the visual world is form. Form is what Torgerson (1958) would call a "complex attribute"; it is capable of variation along an array of dimensions. Brown and Owen (1967) have demonstrated, for sets of random shapes of varying complexity, that the more complex the set of forms, the greater the number of orthogonal physical dimensions needed to describe that set adequately. If a set of forms can be characterized by specifying the value each assumes on a number of physical dimensions, it then becomes meaningful to ask to what extent people (Ss) use these dimensions of physical stimulus variation in making similarity judgments about forms.

If, in the case of form perception, a population of potentially relevant physical dimensions is assumed and if that population is relatively large, then individual $\mathrm{Ss}$ would necessarily use only some of the dimensions to characterize the forms. Each $S$ would select a sample of the dimensions (cues) and respond in terms of that sample. Different Ss might choose different samples of cues, and therefore, individual differences in similarity judgments would result. Since domains of greater complexity would require a larger population of physical dimensions, greater variability

*The work reported here was carried out while the author was a USPHS trainee in Measurement Psychology at the University of Illinois at Urbana-Champaign. The author wishes to ack nowledge the encouragement assistance, and support of his thesis advisor, Dr. Ledyard $R$ Tucker. Other members of the thesis committee were Drs. Hake, Osgood, Batchelder, N. Wiggins, Burkholder, and Hohn, and their guidance was greatly appreciated. in the sample of dimensions would be expected to result. One must therefore ask in a form perception task what sorts of differences exist between Ss in the dimensions used to make judgments and the manner in which those dimensions are used.

Tucker and Messick (1963) have developed a model for multidimensional scaling which permits the isolation of individual differences in perceptual judgments. Consistent individual differences about a psychological domain can be detected, and a separate multidimensional spatial model can be constructed for each viewpoint. Helm and Tucker (1962) have demonstrated the utility of this method for investigating individual differences in color perception, and Tucker and Messick (1963) have used it to isolate consistent individual viewpoints about the similarity of political figures. Methodological discussions of the Tucker-Messick procedure may be found in Tucker and Messick (1963), Tucker (1966), Wiggins and Pradhan (1963), Ross (1966), and Cliff (1968).

Little published work has been explicitly concerned with individual differences in form perception. Studies by Brown and his co-workers (Behrman and Brown, 1968; Brown and Andrews, 1968), using four-turn shapes, revealed only minimal individual differences. A multidimensional scaling study of form perception explicitly concerned with individual differences was reported by Silver, Landis, and Messick (1966). They required 50 Ss to judge the pairwise similarities of 30 forms which varied in complexity. Five distinct viewpoints of varying dimensions of the viewpoints were dimensionalities were isolated. The then related to four physical measures of the forms: dispersion $\left(P^{2} / A\right)$, angular variability, complexity, and rotation.

The present investigation was designed to explore the dimensions of variation in Ss' similarity judgments of a certain class of complex forms and to determine the extent of individual differences in such judgments. The basic experimental task required Ss to evaluate the similarity of pairs of stimuli. The stimuli were random forms which had previously been characterized on a collection of physical measures.

By analyzing Ss' judgments of stimulus similarity, it is possible to determine if there are consistent differences in the way individuals perceive the forms. For each $S$ type, a geometric model of his perceptual space can be derived using multidimensional scaling techniques. These models can, in turn, be related to the physical characteristics of the stimuli.

The present study differs from that of Silver, Landis, and Messick in that it includes (1) the selection of stimuli from a clearly defined stimulus population; (2) the selection of stimuli in terms of a specific set of physical measures, so that the salience of that physical characterization could be assessed; (3) the inclusion of a broader set of basic form measures, and (4) the use of Kruskal's (1964a, b) computer program for multidimensional scaling (M DSCAL-Version 3). Kruskal's general approach to scaling represents an elaboration and refinement of ideas originally developed by Shepard (1962a, b).

\section{METHOD \\ Subjects}

Forty-nine persons served as Ss for this investigation. Of these, 27 were enrolled in an introductory psychology course and received credit for their participation. The other 22 were volunteers who were paid for their services. Forty-six Ss were University of Illinois undergraduates, one was a graduate student, and two were nonstudents. Seventeen Ss were male, 32 female.

\section{Stimuli}

Twenty-one random forms were selected from the set of 200 12-turn shapes constructed by Brown and Owen (1967). These forms had been generated by Method 1 of Attneave and Arnoult (1956) and were roughly equated for area. The forms were chosen as markers on the five most prominent factors isolated by Brown and Owen for physically describing 12-turn shapes. Factor scores on all 12 Brown and Owen dimensions were 
included in the characterization of each form for the various analyses.

Seventeen physical measures of the type previously used by Attneave (1957), Stenson (1966), and Silver, Landis, and Messick (1965) were also taken on each form. Basic measures of this type were used to derive the factors proposed by Brown and Owen. The 17 basic physical measures were:

(1) P. The length of the perimeter as measured in millimeters; the sum of all the side lengths.

(2) $A$. The area enclosed by the perimeter of the form as measured in square millimeters.

(3) $P^{2} / A$. The ratio of the square of the perimeter to the area. Attneave (1957) proposed this as a measure of dispersion or noncompactness.

(4) VE/HE. The ratio of vertical to horizontal extent of the form. This measure was used by Brown and Andrews (1968) as a measure of rotation.

(5) VIA. The variance of the interior angles of the form. The angles were measured in degrees.

(6) MDIA. The mean of the differences between successive interior angles, measured in degrees.

(7) VDIA. The variance of the differences between successive interior angles.

(8) MSA. The mean of the angles in the form, when angles are measured so that the value obtained is less than 180 deg. Thus for this measure (as well as the next three), either the interior or the corresponding exterior angle was measured, depending upon which was smaller.

(9) VSA. The variance of the angles measured as values less than $180 \mathrm{deg}$.

(10) MDSA. The mean of the differences between- successive angles when angles are measured as in (8).

(11) VDSA. The variance of the differences between successive angles when angles are measured as in (8).

(12) ROTS. Rotation measured in degrees by the deviation of the line of greatest extent from the vertical in either a clockwise or counterclockwise direction. The line of greatest extent is drawn through the two most widely separated points on the perimeter of the form.

(13) ROTO. Rotation measured in degrees by the deviation of the line of greatest extent from the vertical in a clockwise direction. This measure is similar to what Stenson (1966) called orientation.

(14) HE. The horizontal extent of the form as measured in millimeters.

(15) VE. The vertical extent of the form in millimeters.

(16) LGE. The length, in millimeters, of the line connecting the two most distant points on the perimeter of the form.
(17) SBRT. A subjective measure of rotation. The orientation of the form was judged by $E$ in terms of the LGE and measured from the vertical in degrees.

The matrix of intercorrelations between the basic physical measures revealed a high correlation between $P$ and $P^{2} / A(.72)$, suggesting that the attempt to equate for area was largely successful. Area was respectably uncorrelated with all other measures except $\mathbf{P}^{2}$ A'. VIA, MDIA, VDIA, and MSA, all angle measures, formed a cluster of highly correlated items. MSA and MDIA could reasonably have been discarded from the set of physical measures. $P$ was correlated with three of these angle measures: VIA, MDIA, and MSA. Another cluster of correlated angle measures involved VSA, MDSA, and VDSA. The four measures presumed to indicate rotation (VE/HE, ROTS, ROTO, SBRT) were sufficiently uncorrelated with each other to justify the inclusion of all four. VE/HE was, of course, correlated with both $\mathrm{HE}$ and VE.

Correlations of the Brown and Owen dimensions and these physical measures supported Brown's interpretation of Dimensions I and II as compactness and jaggedness, although Dimension $I$ is not free of the influence of area. None of the physical measures included here seem appropriate to reflect what Brown and Owen interpret as $x^{-}$and $y$-axis asymmetry (Dimensions III and IV). Dimension $V$ is labeled rotation by Brown but does not correlate significantly with any of the four physical measures assumed to reflect rotation. Since the four rotation measures did not correlate with each other, one can assume that five separate ideas of rotation are included here. Brown and Owen (B \& O) Dimension $V / I$ is, however, related to VE/HE. B \& O VIII involves dispersion and angularity, and $B \& O$ XI correlates with measures which involve both the internal and external angles of the form (VSA, MDSA, VDSA). The other Brown and Owen dimensions did not relate to any of these basic physical measures.

\section{Scaling Task}

Photographs of the forms were mounted in pairs on $8 \times 5$ in. index cards, and each card was enclosed in clear plastic. The forms were distributed so as to appear equally often on the right and left sides of the cards. Two hundred and ten of the cards contained all possible pairings of two distinct forms; 21 cards contained pairs of identical forms. Thus, a total of 231 stimulus cards, each containing two forms, constituted the set of objects to be considered by the Ss.
Judgments of the similarity between the pairs of forms were obtained by a variant of the method of graded dichotomies (Richards, 1971). The set of 231 cards was distributed randomly into eight piles. Through a series of pairwise comparisons of the piles, Ss imposed an ordering on the piles with respect to the similarity between the forms on each of the cards. That is, Ss partitioned the set of 231 cards into eight categories or levels of similarity and ordered those eight levels from most to least similar. All Ss required about $2 \mathrm{~h}$ to complete the scaling task and to verify the result.

The Ss' judgments placed each stimulus pair into one of eight categories ordered from most to least similar. For all Ss, the most similar pile contained the 21 identity pairs, and many Ss placed additional cards into this pile. The values 0-7 were assigned to the piles, ranging from most to least similar, respectively. These values entered into a 49 by 231 matrix of Ss by Stimulus Pairs $(=\mathrm{X})$. Each entry represents a judged distance or proximity measure between the two stimuli in the given pair for each $S$.

A variation of the Tucker-Messick (1963) procedure for isolating individual differences in perceptual judgments was followed. The matrix of cross products between Ss over stimulus pairs was formed, multiplied by a scaling constant, and then factored into its principal components (Nunnally, 1962; Tucker, 1966). Tucker's mean square ratio criterion (Tucker, 1960) for when to stop factoring was used. The first six components all had mean square ratios greater than 2.00 which, when referred to an $F$ distribution, proved to be highly significant. The distribution of roots for components beyond six behaved erratically, and the corresponding mean square ratios were of questionable significance. Therefore, six components were retained for subsequent analyses. These six components are the axes of the space in which $S s$ are embedded. They reflect individual differences in the Ss' judgmental systems. The loadings on them are the basis for describing and differentiating between Ss.

A matrix $Z$, computed from the six largest roots $(\lambda \mathrm{m})$ and their associated characteristic vectors $\left(V_{m}\right)$, contained as entries the projections of Ss on the first six principal components. The six $S$ factors corresponding to the columns of $\mathrm{Z}$ accounted for $91.13 \%$ of the total sums of squares. These six factors were rotated to a Binormamin solution (Kaiser \& Dickman, 1959) in an attempt to approximate oblique simple structure and to pass the 
Table 1

Analysis of Distributions of Interstimulus Distances for the Six Idealized Individuals

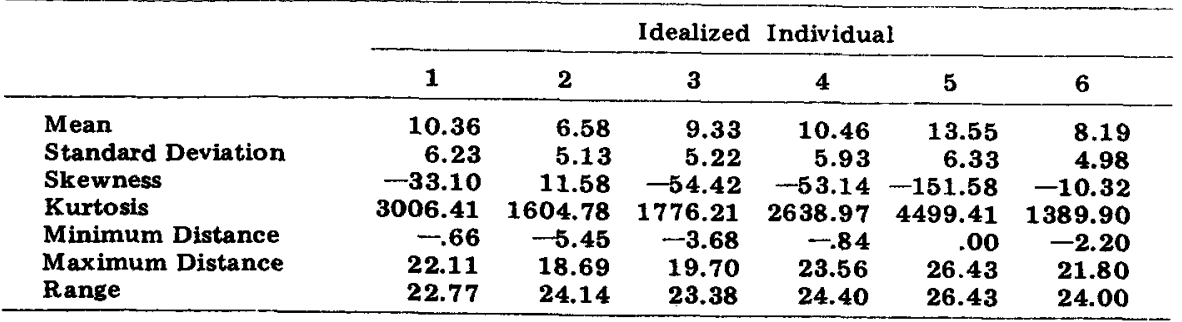

components through clusters of Ss. All six rotated components were essentially unipolar. The six rotated axes of the Binormamin system can be taken as representing "idealized individuals," where an "idealized individual" is defined as a "direction in the factor space resulting from a factor analysis of individuals" (Helm \& Tucker, 1962).

For each idealized individual, a set of interstimulus similarities was derived. From the basic model for principal component analysis, we have $\mathrm{ZB}=\mathrm{X}$, where $\mathrm{X}$ is the obtained data matrix, $\mathrm{Z}$ is the matrix of loadings of Ss on principal components, and $B$ is the matrix of stimulus pair projections on the principal components of the $S$ space. In the present analysis, $B$ was computed from the relation $B^{\prime}=$ $X^{\prime} Z\left(Z^{\prime} Z\right)^{-1}$. The stimulus pair projections on the dimensions corresponding to the Binormamin rotated factors are of interest for scaling purposes. These were obtained by taking $B_{R}=B^{\prime}\left(T^{\prime}\right)^{-1}$, where $T$ is the transformation to structure matrix of the Binormamin rotation. The entries in the six columns of $B_{R}^{\prime}$ were taken as judged similarities between stimulus pairs for the six idealized individuals.

The distribution of judged interstimulus distances for each II is summarized in Table 1 . The differences in the means and skewness reflect differences in the amount of similarity attributed to the stimulus set as a whole. Thus, II2 judged more stimuli to be similar than did II5. II2 is the only viewpoint for which the distribution is positively skewed, indicating a tendency for judgments to pile up at the similar end of the scale. II5 represents the extreme case of negative skewness; this viewpoint involved many form pairs being placed at the dissimilar end of the scale. Other viewpoints were spread between these two extremes with respect to means and skewness. All six distributions were platyk urtic.

Each column of $B_{R}^{\prime}$ was scaled by the Shepard-Kruskal technique using Version 3 of MDSCAL. Thus, separate scaling was done on data for the six idealized individuals. In addition, the raw data of 11 Ss selected as markers for the six IIs were scaled. The results for individual $S$ s are not reported in detail; however, they were used to assess and clarify the idealized individuals.

In all cases, MDSCAL was entered with dissimilarity judgments (high values indicate greater dissimilarity, low values indicate similarity). Initially, 25 iterations were specified and a STRMIN, an acceptable stress level, of .05 was used. Configurations were obtained varying in dimensionality from 10 to 1 . Whenever the stress had not attained a minimum value in 25 iterations, the configuration was reentered and 25 more iterations done with STRMIN set at 0.0.

For the perceptual spaces of the six idealized individuals, configurations ranging from four to seven dimensions seemed plausible in terms of stress levels and the distribution of stress over various dimensionalities. In scaling the raw data from the 11 marker Ss, five dimensions was the modal number indicated by the distribution of stress values. In addition, the forms used in this study were selected in terms of their values on five physical variables (B \& O I-V). Therefore, a five-dimensional Euclidean configuration was obtained for each of the six IIs. The stress levels corresponding to these solutions were $.082, .096, .092, .109, .085$, and .088 for IIs $1-6$, respectively. $i$

For each idealized individual, the configuration obtained from MDSCAL was rotated to a Varimax criterion (Harman, 1967) in an attempt to achieve orthogonal simple structure. Thus, two spatial models were derived from the judgments of stimulus similarities for each idealized individual: the configuration produced by MDSCAL ( $P$ dimensions) and the Varimax rotation of that configuration ( $V$ dimensions).

For each II, matrices of simple correlations were computed relating the two sets of psychological dimensions to the basic physical measures. Presumably, the various physical measures assess aspects of the forms (cues) which Ss could use in making similarity judgments. Each stimulus provides a multiplicity of cues, but any given $S$ will utilize only a few of those cues available to him. Individual differences should be reflected in different patterns of cue utilization. The magnitude of the correlation between a physical dimension and a psychological dimension was assumed to reflect the degree to which the cue influenced that psychological dimension. Coefficients exceeding a value of .50 were taken to reflect use of a cue, and such correlations were used to interpret the psychological dimensions. Tables 2-7 contain these correlations for the six IIs.

\section{Idealized Individual One}

For this II (see Table 2), both P1 and P2 appear to be size-dispersion dimensions: the differences between these dimensions are reflected in the angle measures (MDIA, VDIA, MSA, VSA, MDSA, VDSA) and two of the

Table 2

Correlations of the Psychological Dimensions of Idealized Individual 1 with the Basic Physical Measures

\begin{tabular}{|c|c|c|c|c|c|c|c|c|c|c|}
\hline \multirow{3}{*}{$\begin{array}{c}\text { Basic } \\
\text { Physical } \\
\text { Measures }\end{array}$} & \multicolumn{10}{|c|}{ Psychological Dimensions } \\
\hline & \multicolumn{5}{|c|}{ Unrotated } & \multicolumn{5}{|c|}{ Varimax Rotated } \\
\hline & $\mathbf{P 1}$ & P2 & $\mathbf{P 3}$ & P4 & P5 & $\mathbf{v 1}$ & V2 & V3 & V4 & V5 \\
\hline $\mathbf{P}$ & .17 & .19 & .33 & -.10 & .79 & -.49 & -.36 & .45 & .36 & .32 \\
\hline A & -.62 & -.73 & .05 & .12 & .15 & -.69 & -.20 & -.51 & .16 & -.51 \\
\hline $\mathbf{P}^{2} / \mathbf{A}$ & .50 & .70 & .21 & -.13 & .54 & .05 & -.12 & .66 & .21 & .56 \\
\hline VE/HE & -.01 & -.02 & -.34 & -.07 & .14 & -.15 & -.28 & -.25 & .03 & .21 \\
\hline VIA & .28 & .26 & .28 & -.54 & .43 & -.25 & -.24 & .57 & -.26 & .30 \\
\hline MDIA & .30 & .12 & .25 & -.61 & .51 & -.32 & -.36 & .53 & -.27 & .39 \\
\hline VDIA & -.17 & -.07 & .12 & -.21 & .09 & -.29 & -.02 & .04 & -.17 & -.14 \\
\hline MSA & -.32 & -.18 & -.35 & .52 & -.37 & .21 & .29 & -.60 & .26 & -.21 \\
\hline VSA & -.21 & .24 & -.26 & -.07 & .16 & -.21 & .19 & -.18 & .02 & .29 \\
\hline MDSA & .02 & .32 & -.13 & .02 & .25 & -.07 & .04 & -.01 & .15 & .33 \\
\hline VDSA & -.22 & .31 & -.32 & -.23 & .01 & -.14 & .32 & -.13 & -.23 & .27 \\
\hline ROTS & .03 & -.13 & -.38 & .13 & -.11 & .14 & -.09 & -.26 & .08 & .10 \\
\hline ROTO & .26 & .00 & .34 & -.28 & -.27 & .21 & -.11 & .34 & -.38 & -.20 \\
\hline HE & -.06 & -.09 & .48 & .15 & -.06 & -.03 & .18 & .25 & .13 & -.30 \\
\hline VE & -.08 & -.13 & -.08 & .01 & .20 & -.32 & -.33 & -.17 & .17 & .05 \\
\hline LGE & -.22 & -.24 & .30 & $\mathbf{. 3 3}$ & .04 & -.16 & -.09 & -.25 & .30 & -.33 \\
\hline SBRT & .07 & .05 & .11 & -.24 & .10 & .09 & -.04 & .06 & -.32 & -.04 \\
\hline
\end{tabular}


Table 3

Correlations of the Psychological Dimensions of Idealized Individual 2 With the Basic Physical Measures

Psychological Dimensions

\begin{tabular}{|c|c|c|c|c|c|c|c|c|c|c|}
\hline \multirow{2}{*}{$\begin{array}{c}\text { Basic } \\
\text { Physical } \\
\text { Measures }\end{array}$} & \multicolumn{5}{|c|}{ Unrotated } & \multicolumn{5}{|c|}{ Varimax Rotated } \\
\hline & P1 & P2 & P3 & P4 & P5 & V1 & V2 & V3 & V4 & V5 \\
\hline $\mathbf{P}$ & .24 & .31 & .46 & .46 & .09 & -.04 & .33 & .49 & .42 & -.20 \\
\hline A & -.28 & -.58 & -.05 & .54 & -.50 & -.01 & -.35 & -.43 & .32 & -.71 \\
\hline$P^{2} / A$ & .40 & .61 & .46 & .00 & .39 & -.04 & .47 & .72 & .11 & .28 \\
\hline $\mathrm{VE} / \mathrm{HE}$ & .36 & .02 & -.24 & .27 & -.32 & .56 & .06 & -.02 & .12 & -.23 \\
\hline VIA & -.09 & .57 & .37 & .20 & .06 & --.26 & .60 & .29 & .13 & -.07 \\
\hline MDIA & -.18 & .41 & .25 & .26 & .08 & -.27 & .47 & .13 & .21 & -.07 \\
\hline VDIA & -.38 & .33 & .38 & .18 & -.42 & -.35 & .47 & -.01 & -.09 & -.51 \\
\hline MSA & .16 & -.49 & -.27 & -.22 & -.07 & .26 & -.54 & -.16 & -.16 & .05 \\
\hline VSA & .30 & .14 & .30 & -.07 & -.04 & .08 & .07 & .41 & -.09 & -.06 \\
\hline MDSA & .34 & .09 & .37 & -.14 & .19 & -02 & -.04 & .52 & -.04 & .11 \\
\hline VDSA & .14 & .04 & .09 & -.30 & .21 & -.04 & -.08 & .21 & -.18 & .27 \\
\hline ROTS & -.20 & .15 & -.15 & .29 & -.01 & -.03 & .25 & -.22 & .24 & -.05 \\
\hline ROTO & .14 & -.06 & -.27 & -.32 & .00 & .23 & -.14 & -.09 & -.26 & .22 \\
\hline HE & -.24 & -.13 & .08 & -.12 & .27 &.$- \mathbf{3 3}$ & -.15 & -.05 & .01 & .18 \\
\hline VE & .44 & -.07 & -.35 & .38 & -.31 & 69 & -.02 & -.08 & .24 & -.23 \\
\hline LGE & .20 & -.43 & -.04 & -.13 & -.28 & .24 & -.45 & -.02 & -.18 & -.22 \\
\hline SBRT & -.18 & -.35 & .30 & -.59 & -.01 & -.40 & -.44 & .09 & -.52 & .03 \\
\hline
\end{tabular}

rotation measures (ROTS, ROTO). P2 is quite strikingly related to $B$ \& $O$ I ( $\mathrm{r}=-.81, \mathrm{p}<.001$ ), while $\mathrm{P1}$ shows no promising correlations with any of the $B \& O$ measures. P3 is marginally related to horizontal extent $(r=.48$, $\mathrm{p}<.05) . \mathrm{P} 4$ involves the angularity or jaggedness aspect of the forms (as measured by VIA, MDIA, MSA). The fifth $\mathrm{P}$ dimension, like $\mathrm{P} 1$ and $P 2$, is a dispersion dimension; however, $\mathrm{P}_{5}$ is more strongly related to the length of the perimeter than to the area (A) of the form.

The Varimax-rotated perceptual space contains three interpretable dimensions. $V 1$ is a size dimension, V5 is size dispersion, and V3 is a combination of angularity and dispersion. V2 and V4 show no significant correspondence with any of the 17 physical measures. The 17 physical measures provide interpretations for four $P$ dimensions and a possible (suggestive) interpretation of the fifth. Only three $\mathrm{V}$ dimensions are clearly interpretable in terms of the basic physical measures, and these are redundant. Size, dispersion, and angularity appear to be the most useful physical cues for this II.

\section{Idealized Individual Two}

The 17 physical measures are related to the two psychological spaces of 112 in Table 3 . P2 is strongly related to $A, P^{2} / A$, VIA, and marginally related to MSA. It would seem to be basically a size-dispersion dimension. The cues influencing $\mathbf{P 4}$ are $A$, area, and SBRT, the subjectively assessed rotation index. $P 5$ relates to $A$, and $P 3$ is marginally related to both $P$ and $\mathbf{P}^{2} / \mathrm{A}$. Dispersion, size, and rotation were important cues for this II, but their influence was redundantly distributed over dimensions of the $P$ space.
The $\mathbf{V}$ dimensions provide a much richer, but more nicely interpretable, picture. V1 is related strongly to VE, the elongation of the form along the $y$-axis, and to VE/HE, the discrepancy or uniformity of the $x$ - and $y$-axis elongations. The two significant and several marginal correlations of physical dimensions with V2 suggest that this dimension is angularity, with primary importance given to the interior angles of the form. V3 is clearly compactness dispersion, V4 is rotation (subjectively assessed), and $V 5$ is area or size. Thus, the V space is preferable to the $P$ space in relation to the basic physical measures. The cues used by this II were vertical extent, angularity, dispersion, rotation, and area.

\section{Idealized Individual Three}

Three $P$ dimensions were interpretable in terms of the basic physical measures, as is apparent from
Table 4. P2 is significantly correlated with MSA and has marginal correlations with VIA, MDIA, and VDIA, thus suggesting an angularity dimension. P3 is clearly rotation. As for II2, SBRT is the important indicator of the rotation of the form. $P 5$ is compactness dispersion, since it correlates with both $\mathrm{P}$ and $\mathrm{P}^{2} / \mathrm{A}$.

The $\mathrm{V}$ dimensions involved a greater number of significant relationships with the physical measures, but again, only three psychological dimensions were interpretable. V2 is clearly rotation as measured by SBRT and by $\mathrm{VE} / \mathrm{HE}$. V4 is an angle dimension; however, it is different from the angularity dimensions of IIs 1 and 2. The previous angularity dimensions involved basically measures on the interior angles of the form. Here two of the measures (MSA, VDSA) deal with both interior and exterior angles. In addition, two of the measures involve the variance of the difference between successive angles (VDIA, VDSA). A plausible interpretation of V4 is that it reflects the uniformity of the angles of the form. V5 is a conglomerate of dispersion and angularity (in the previous sense as with IIs 1 and 2). A fourth dimension, $V 3$, is marginally related to $P$ and $P^{2} / A$, but it is partially redundant with V5. V1 has no significant correlations with any of the basic physical measures. Thus, in terms of relating to the physical measures, the $V$ space is the more interesting of the two psychological configurations. This II appears to have used two kinds of angularity cues, in addition to dispersion and rotation.

\section{Idealized Individual Four}

Table 5 contains the correlations between the basic physical measures and the psychological spatial models for II4. $\mathrm{P2}$ is indexed by MDIA and

Table 4

Correlations of the Psychological Dimensions of Idealized Individual 3 With the Basic Physical Measures

\begin{tabular}{|c|c|c|c|c|c|c|c|c|c|c|}
\hline \multirow{3}{*}{$\begin{array}{c}\text { Basic } \\
\text { Physical } \\
\text { Measures }\end{array}$} & \multicolumn{10}{|c|}{ Psychological Dímensions } \\
\hline & \multicolumn{5}{|c|}{ Unrotated } & \multicolumn{5}{|c|}{ Varimax Rotated } \\
\hline & P1 & P2 & $\mathbf{P 3}$ & P4 & P5 & $\mathbf{V 1}$ & $\mathbf{V 2}$ & V3 & $\mathbf{V 4}$ & V5 \\
\hline $\mathbf{P}$ & .24 & .40 & .32 & .32 & .62 & -.15 & -.28 & .46 & .26 & .70 \\
\hline $\mathbf{A}$ & -.13 & .24 & .11 & .07 & -.03 & -.15 & .10 & -.08 & .22 & .11 \\
\hline $\mathbf{P}^{2} / \mathbf{A}$ & .24 & .15 & .23 & .24 & .53 & -.06 & -.27 & .49 & .09 & .47 \\
\hline VE/HE & -.01 & .00 & -.35 & .14 & .38 & -.07 & -.54 & .03 & -.06 & -.02 \\
\hline VIA & .11 & .49 & .37 & .40 & .33 & -.14 & -.06 & .32 & .47 & .56 \\
\hline MDIA & .24 & .45 & .42 & .28 & .30 & -.06 & .02 & .31 & .36 & .64 \\
\hline VDIA & -.33 & .45 & .31 & .34 & -.05 & -.32 & .20 & .03 & .60 & .15 \\
\hline MSA & -.13 & -.54 & -.43 & -.40 & -.26 & .11 & -.03 & -.28 & -.53 & -.60 \\
\hline VSA & .01 & -.27 & -.24 & -.07 & .26 & -.01 &.- .33 & .13 & -.30 & -.17 \\
\hline MDSA & .18 & -.22 & -.02 & -.16 & .41 & -.02 & -.26 & .30 &.- .37 & .14 \\
\hline VDSA & .04 & $-.2 \overline{2}$ & -.23 & -.34 & .11 & .03 & -.15 & -.03 & -.50 & -.18 \\
\hline ROTS & -.02 & .16 & -.18 & .22 & -.10 & .09 & -.12 & -.17 & .23 & -.09 \\
\hline ROTO & .13 & .02 & .11 & -.09 & -.14 & .11 & .19 & -.07 & -.01 & .12 \\
\hline HE & .18 & -.01 & .35 & -.07 & -.35 & .20 & .49 & .01 & .09 & .12 \\
\hline VE & .17 & .00 & -.28 & .21 & .31 & .10 & -.48 & .05 & .01 & .09 \\
\hline LGE & -.15 & -.34 & .16 & -.18 & .22 & -.26 & .03 & .22 & -.20 & .03 \\
\hline SBRT & .05 & -.06 & .62 & -.42 & -.16 & -.10 & .68 & .20 & -.20 & .25 \\
\hline
\end{tabular}


MSA, with marginal relations to $A$, VIA, and VE, and appears to be an angularity dimension. However, this dimension only partially overlaps with angularity as discussed above (IIs 1 and 2). P4 correlates significantly with VSA, and P5 with $P$, the length of the perimeter. This II seems to have used some aspects of angularity and dispersion in judging the forms.

In the rotated configuration, $\mathrm{V} 2$ is related to $P, V 4$ is relatively strongly correlated with vertical extent, and V5 is related to MDIA-the mean difference between successive interior angles. V3 is indexed by VIA, MSA, and VSA and is thus an angularity dimension, primarily influenced by the small (either interior or exterior) angles of the form. The Varimax-rotated configuration thus has four interpretable dimensions and is, therefore, preferred to the unrotated space. Several angle cues, vertical extent, and the length of the perimeter were important for II4.

\section{Idealized Individual Five}

The basic physical measures provide interpretations for three $\mathbf{P}$ dimensions, as may be seen in Table 6. P2 is indexed by VIA, MDIA, and MSA and is thus an angularity dimension similar to that of II1. P4 relates significantly to VDSA, a measure of the uniformity of the small angles of the forms. P5 appears to be a combination of dispersion $\left(P, P^{2} / A\right)$ and angularity (VIA, MDIA, MSA). V5 is very similar to $\mathrm{P5}$ and has high correlations with $\mathrm{P}$, VIA, MDIA, and MSA. V1 is related only to MDIA-the mean difference between successive internal angles of the forms. V3 relates most strongly to MSA, somewhat to VIA, and marginally to ROTO. Neither psychological configuration receives a set of simple, nonredundant interpretations in terms of the basic physical measures. Angularity and dispersion cues, however, were certainly used by this II.

\section{Idealized Individual Six}

In Table 7, P5 is significantly correlated with VIA, MDIA, and MSA and is thus angularity in the usual sense. P1 through $\mathrm{P} 4$ do not relate, even marginally, to any of the basic physical measures. In the rotated configuration, V5 is angularity and is essentially the same as $\mathrm{P} 5$. V1 appears to be size dispersion, and $\mathrm{V2}$ is marginally related to $\mathrm{VE}$ and $\mathrm{VE} / \mathrm{HE}$, suggesting that elongation along the $y$-axis may have been important for II6.

Are the IIs Really Different?

Table 8 summarizes the use of the physical cues by the IIs. The entry $P$ means that the particular II used the indicated cue (in the correlational
Table 5

Correlations of the Psychological Dimensions of Idealized Individual 4 With the Basic Physical Measures

Psychological Dimensions

\begin{tabular}{|c|c|c|c|c|c|c|c|c|c|c|}
\hline \multirow{2}{*}{$\begin{array}{c}\text { Basic } \\
\text { Physical } \\
\text { Measures }\end{array}$} & \multicolumn{5}{|c|}{ Unrotated } & \multicolumn{5}{|c|}{ Varimax Rotated } \\
\hline & P1 & P 2 & P 3 & P4 & P5 & V1 & $\mathbf{V 2}$ & V3 & V4 & $\mathbf{V 5}$ \\
\hline $\mathbf{P}$ & .31 & .13 & .21 & .42 & .56 & -.02 & .51 & .24 & .12 & .49 \\
\hline $\mathbf{A}$ & .21 & -.46 & -.07 & .13 & .29 & -.41 & .36 & -.23 & .29 & .15 \\
\hline $\mathbf{P}^{2} / \mathrm{A}$ & .00 & .35 & .26 & .32 & .24 & .20 & .16 & .44 & -.04 & .21 \\
\hline VE/HE & .20 & -.40 & .14 & -.02 & -.23 & -.21 & -.17 & -.14 & .41 & .08 \\
\hline VIA & -.08 & .49 & .43 & .15 & .32 & .15 & .06 & .52 & -.30 & .38 \\
\hline MDIA & .20 & .52 & .32 & .18 & .43 & .23 & .26 & .35 & -.24 & .54 \\
\hline VDIA & -.45 & .06 & .37 & .00 & .14 & -.26 & -.14 & .42 & -.24 & .01 \\
\hline MSA & .12 & -.53 & -.45 & -.28 & -.27 & -.19 & -.10 & -.64 & .24 & -.33 \\
\hline VSA & .27 & -.22 & -.17 & -.56 & .17 & -.20 & -.10 & -.62 & -.15 & .19 \\
\hline MDSA & .22 & -.08 & -.02 & -.37 & .22 & -.13 & -.05 & -.36 & .15 & .27 \\
\hline VDSA & -.03 & .01 & -.35 & -.34 & .11 & -.01 & .03 & -.39 & -.31 & -.13 \\
\hline ROTS & .24 & -.01 & .13 & .07 & -.25 & .12 & -.09 & .02 & .24 & .15 \\
\hline ROTO & -.38 & .10 & .08 & .04 & -.32 & .08 & -.28 & .29 & -.07 & -.32 \\
\hline HE & -.01 & .25 & -.19 & .28 & .21 & .19 & .38 & .12 & -.09 & -.05 \\
\hline $\mathbf{V E}$ & .37 & -.45 & .03 & .27 & -.21 & -.14 & .09 & -.13 & .65 & .08 \\
\hline LGE & -.12 & -.38 & .24 & .17 & -.04 & -.35 & -.07 & .17 & .32 & -.04 \\
\hline SBRT & -.39 & .04 & .11 & .00 & .18 & -.20 & .00 & .24 & -.25 & -.11 \\
\hline
\end{tabular}

Table 6

Correlations of the Psychological Dimensions of Idealized Individual 5 With the Basic Physical Measures

Psychological Dimensions

\begin{tabular}{|c|c|c|c|c|c|c|c|c|c|c|}
\hline \multirow{2}{*}{$\begin{array}{c}\text { Basic } \\
\text { Physical } \\
\text { Measures }\end{array}$} & \multicolumn{5}{|c|}{ Unrotated } & \multicolumn{5}{|c|}{ Varimax Rotated } \\
\hline & P1 & P2 & $\mathbf{P 3}$ & P4 & P5 & V1 & V2 & V3 & V4 & V5 \\
\hline $\mathbf{P}$ & .07 & .34 & .10 & .11 & .62 & .37 & .08 & .01 & -.04 & .56 \\
\hline A & -.46 & .05 & -.29 & .32 & .06 & .04 & .36 & -.32 & .35 & .08 \\
\hline $\mathbf{P}^{2} / \mathbf{A}$ & .27 & .23 & .32 & -.05 & .45 & .20 & -.15 & .22 & -.18 & .43 \\
\hline VE/HE & -.18 & -.11 & -.02 & .03 & .13 & -.05 & .06 &.- .26 & -.01 & .20 \\
\hline VIA & .28 & .58 & .47 & -.29 & .50 & .31 & .17 & .50 &.- .42 & .61 \\
\hline MDIA & .32 & .67 & .22 & -.35 & .64 & .57 & .28 & .37 & -.47 & .61 \\
\hline VDIA & -.17 & .38 & .42 & .02 & .23 & -.02 & .24 & .33 & .03 & .49 \\
\hline MSA & -.28 & -.69 & -.48 & .21 & -.46 & -.37 & .19 & -.59 & .34 & -.62 \\
\hline VSA & -.01 & -.48 & -.16 & -.25 & .09 & -.20 & -.10 & -.44 & -.31 & -.16 \\
\hline MDSA & -.03 & -.39 & .07 & -.19 & .17 & -.25 & -.16 & $-\mathbf{3 0}$ & -.25 & .04 \\
\hline VDSA & -.07 & -.34 & -.10 & -.57 & -.14 & -.31 & .12 & -.17 & -.39 & -.34 \\
\hline ROTS & -.24 & .15 & -.05 & .03 & .05 & .08 & .21 & -.08 & .21 & .21 \\
\hline ROTO & .41 & .24 & .18 & .01 & -.25 & .14 & -.17 & .49 & -.13 & -.13 \\
\hline $\mathbf{H E}$ & .17 & .21 & -.16 & .14 & -.16 & .23 & -.02 & .21 & .18 & -.21 \\
\hline VE & -.11 & .05 & -.22 & .23 & .07 & .20 & .09 & -.23 & .17 & .12 \\
\hline LGE & -.24 & -.07 & .12 & .34 & .01 & -.17 & -.05 & -.08 & .29 & .16 \\
\hline SBRT & .06 & .10 & .22 & -.16 & -.03 & -.07 & .00 & .25 & -.09 & .04 \\
\hline
\end{tabular}

Table 7

Correlations of the Poychological Dimentions of Idealized Individual 6 With the Basic Physicul Measures

Psychological Dimensions

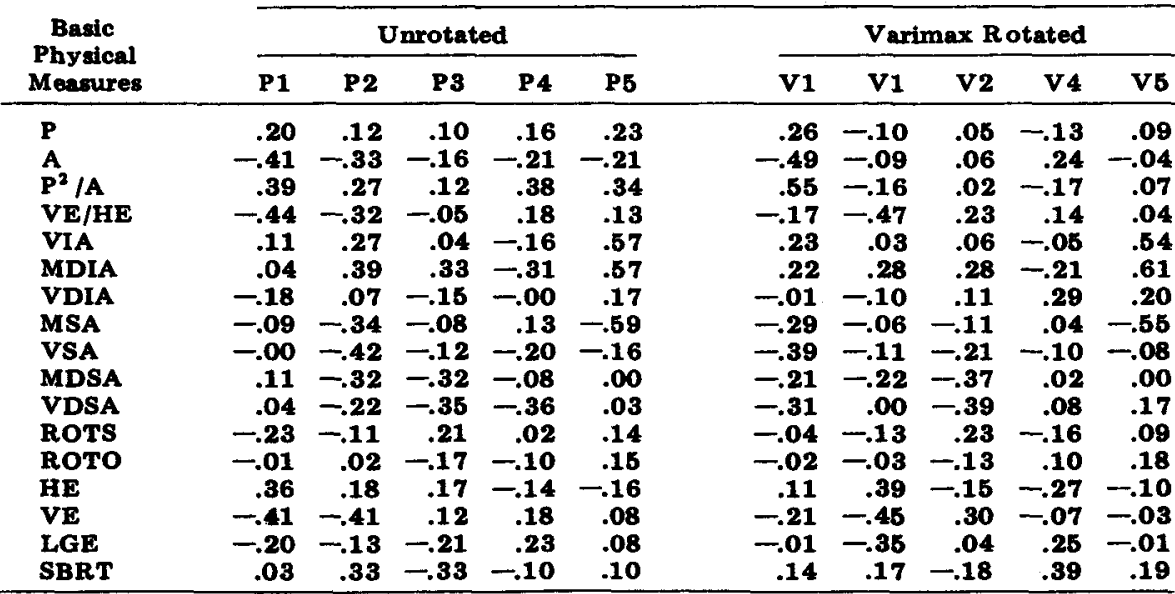


Table 8

Use of the Physical Cues by the Six Idealized Individuals

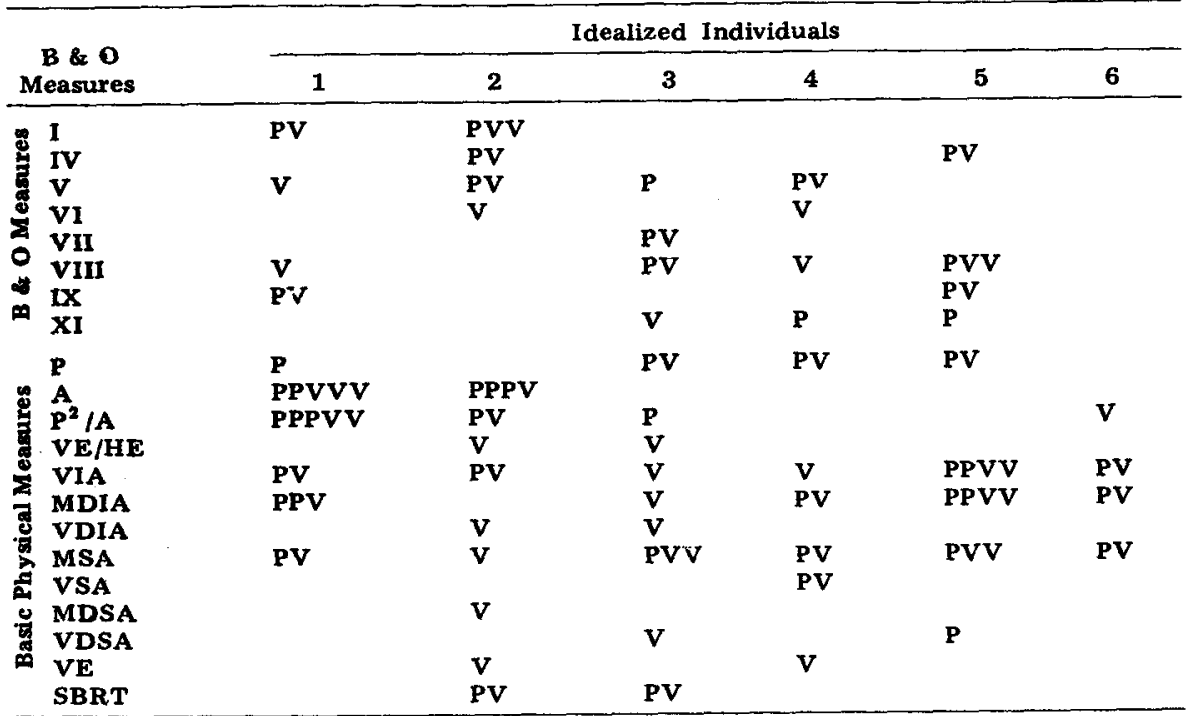

sense discussed above) in his unrotated psychological space ( $P$ dimension), and the entry $V$ indicates the same thing for the rotated psychological space (V dimension). The number of repeated letters shows how many such dimensions correlated significantly with that particular cue for each II. Thus, for example, II1 used area (A) as a cue for two $P$ dimensions and three $\mathrm{V}$ dimensions.

Several features of Table 8 should be noted. Four Brown and Owen measures (B \& $O$ II, III, $X$, and XII) and four basic physical measures (ROTS, ROTO, HE, and LGE) do not appear in the table because none of the psychological dimensions of any II correlated with them. Differences are evident between the six IIs in their pattern of utilization of the remaining eight B \& $O$ cues. II6 used none of the $B \& O$ cues and appeared to be operating independently of the physical properties of the forms. IIs 1 and 2 used $B \& O \quad I$, the compactness-dispersion dimension. IIs 2 and 5 used $B$ \& $O$ IV, skewness along the $\mathrm{x}$-axis; and IIs $1,2,3$, and 4 all used $B$ \& $O V$ (rotation) to some extent. A table could be constructed involving the eight used $B$ \& $O$ measures: an $X$ would be entered whenever a cue had been used and an $O$ elsewhere. Then each II would display a unique pattern of $\mathrm{Xs}$ and $\mathrm{Os}$ and thus a unique pattern of cue utilization for the $B$ \& $O$ dimensions.

A similar differentiation of the IIs can be done using the 13 remaining basic physical measures. VIA and MSA were used, at least to some extent, by all IIs; the other 12 measures differentiate between the IIs. Of particular interest are the measures, $P$, $A$, and $P^{2} / A$-indications of the size and dispersion of the forms. The pattern of use of these three except for 4 and 5 . Area is important only to IIs 1 and 2, while four IIs take advantage of the length of the perimeter and four IIs (not the same

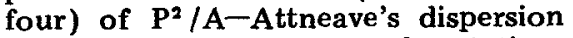
index. Two measures of rotation, VE/HE and SBRT, are important to IIs 2 and 3 , but to none of the others. VE was a cue for IIs 2 and 4 on one rotated dimension. None of the rotation measures in the basic set captures what Brown means by rotation ( $\mathrm{B} \& \mathrm{O} \mathrm{V})$.

The pattern of use of the angle measures also allows differentiation between the IIs. Several different aspects of angularity are obviously represented by these measures. All IIs made use of some angle cues, and this is to be contrasted with the apparent lack of use of B \& O II, said to measure the angularity of the forms.

If further rotations of the psychological spaces were performed, it would be desirable to maintain the high correlations between physical measures and psychological dimensions. The differing patterns of such correlations suggest tha maximizing the interpretability of these spatial models would yield clearly different spaces for the six IIs. In those cases where the IIs have dimensions that appear to overlap, the remaining dimensions of importance are radically different.

Table 9 collects together multiple correlation coefficients relating the dimensions of the unrotated psychological spaces (as predictors) to the five primary $B$ \& $O$ dimensions in constant over $S$ types, and thus measures differs for all pairs of IIs turn. The $B \& O$ dimensions are
between-II comparisons can be made in terms of them. The multiple Rs capitalize upon the redundancies in the psychological spaces and indicate the maximum use of a particular cue. These values take account of the possibility of the influence of a physical cue being spread over several dimensions of the psychological space of a given II. Table 9 again affirms the use of $B \& O$ I by IIs 1 and 2 . IIs 2 and 3 use B \& O II to some extent. II2 clearly relies on B \& O IV, and II4 uses rotation (B \& $O$ V). II6 operates independently of these five physical cues. The differences in cue utilization evident in this table argue most strongly for the separability of the six idealized individuals.

\section{CONCLUSIONS AND DISCUSSION}

Individual differences are clearly evident in the kind of similarity judgments Ss were required to make about this set of forms. Several findings support this assertion: (1) at least six components were necessary to account for the variation between Ss in the scaling data; (2) differences are apparent in the distributions of judged interstimulus distances for the six IIs (Table 1); (3) different patterns of cue utilization were visible for the six IIs (Table 8); and (4) the extent of use of the five $B$ \& $O$ dimensions varied over IIs (Table 9).

The work by Brown and Andrews (1968) and Behrman and Brown (1968) revealed minimal individual differences in similarity judgments about four-turn shapes. However, Silver, Landis, and Messick (1966) found substantial individual differences (five distinct viewpoints) when Ss judged stimuli which varied in complexity. Extensive between-S variation was also evident in the scaling data for the present study; six idealized individuals were isolated. The forms used here, 12-turn shapes, were more complex than those in the two Brown experiments and more so than most of those used by Silver, Landis, and Messick. Increasing the complexity of a stimulus domain

Table 9

Summary of the Multiple Correlation Coefficients for Predicting B \& $O$ Measures from the Psychological Dimensions of each of the Six Idealized Individuals

\begin{tabular}{llllll}
\hline & \multicolumn{5}{c}{ B \& 0 Measures } \\
\cline { 2 - 6 } & I & II & III & IV & V \\
\hline II 1 & .84 & .58 & .37 & .36 & .45 \\
II 2 & .83 & .65 & .51 & .71 & .69 \\
II 3 & .51 & .63 & .35 & .33 & .62 \\
II 4 & .46 & .36 & .36 & .39 & .73 \\
II 5 & .44 & .42 & .34 & .64 & .66 \\
II 6 & .45 & .41 & .36 & .48 & .26 \\
APS & .88 & .40 & .47 & .50 & .74
\end{tabular}


should, and apparently does, increase the extent of individual differences in dealing with that domain.

\section{The Psychophysics}

of Random Forms

In light of this study, which variables seem most useful for characterizing random forms? The dimensions proposed by Brown and Owen (1967) did relate to the dimensions of the psychological spaces and can therefore be said to display some psychological relevance. However, the Brown and Owen dimensions are insufficiently understood to be useful for interpreting the psychological dimensions. Rather, both the $B$ \& $O$ dimensions and the peychological dimensions had to be interpreted in terms of the basic physical measures. The 17 basic physical measures employed here permitted interpretation of $\mathrm{B} \& \mathrm{O}$ dimensions $\mathrm{I}$, II, VI, VIII, IX, and XI; the other six dimensions were not clearly related to the basic physical measures. Four $B$ \& $O$ dimensions (II, III, $X$, and XII) had no direct psychological relevance; the other dimensions did appear to reflect something psychologically meaningful. The $B$ \& $O$ dimensions will, however, only be useful for interpreting psychological dimensions when a well-worked-out set of physical interpretations of them is provided.

The basic physical measures enabled most of the peychological dimensions to be interpreted. The most useful of the 17 physical measures were $P, A$, $P^{2} / A$, and VIA. VIA is similar to what other investigators have called angular variability. The importance of these measures was also reported by Silver, Landis, and Messick and by Brown et al. Only 4 of the 17 physical measures failed to correlate with any psychological dimensions. These were ROTS, ROTO, HE, and LGE. Both HE and LGE were too restricted in range to allow them much correlation with anything. ROTO and ROTS do not appear to be very interesting or relevant psychologically. SBRT seemed from the beginning to be a more reasonable rotation measure, and, although it reflected E's subjective judgments, it did relate to the psychological dimensions of two IIs. The basic physical measures allowed various separable aspects of "dispersion" and "angularity" to be detected in the spaces of the several IIs. Dispersion and angularity are not unitary concepts, but summary terms for several distinct combinations of physical measures.

\section{REFERENCES}

ATTNEAVE, F. Physical determinants of the judged complexity of shapes. Journal of Experimental Psychology, 1957, 53, 221-227.

ATTNEAVE, F. \& AROULT, M. D. The quantitative study of shape and pattern perception. Psychological Bulletin. 1956. 53, $452-471$.

BEHRMAN, B. W., \& BROWN, D. $R$. Multidimensional scaling of form: A psychophysical analysis. Perception \& Psychophysics, 1968, 4, 19-25.

BROWN D. R. \& ANDREWS $M$. Visual form discrimination: Multidimensional analyses. Perception \& Psychophysics, $1968,3,401-406$.

BROWN, D. R., \& OWEN, D. H. The metrics of visual form: Methodological dyspepsia, Psychological Bulletin, 1967. $68,243-259$

CLIFF, N. The "idealized individual" interpretation of individual differences in multidimensional scalina. Psy chometrika. 1968, 33, 225-232.

HELM, C., \& TUCKER, L. R. Individual differences in the structure of color perception. American Joumal of Psychology, 1962. 75. 437-444.

HARMAN, H. H. Modern factor analysis. Chicago: University of Chicago Press, 1960, 1967.

KAISER, H. F., \& DICKMAN, K. W Analytic determination of common factors. Unpublished manuscript. University of Illinois, 1959.

KRUSKAL, J. B. Multidimensional scaling by optimizing goodness of fit to a nonmetrichypothesis. Psychometrika. 1964a, 29, 1-28.

KRUSKA L, J. B. Nonmetric multidimensional scaling: $A$ numerical method. Psychometrika, 1964b, 29. $115 \cdot 130$.

NUNNALLY, J. The analysis of profile data. Psychological Bulletin, 1962, 59, 311-319.

RICHARDS, L. G. A multidimensional scaling analysis of percetved similarity among complex forms. Unpublished manuscript, University of Virginia, 1871

ROSS, J. A remark on Tucker and Messick's "Points of vew" analysis. Psychometrika, 1966, 31, 27-32.

SILVER, C, A.. LANDIS, D., \& MESSICK S. J. Multidimensional analysis of visual form: An analysis of individual form: An analysis of individual differences. A merican
Poychology, 1966, 74, 62-72.

SHEPARD, R. N. The analysis of proximities: Multidimensional scaling with an unknown distance function: I. Psychometrika, 1962a, 27, 125-140.

SHEPARD, R. N. The analysis of proximities: Multidimensional scaling with an unknown distance function: II. Psy chome trik a, $1962 b, 27,219-246$.

STENSON, H. H. The physical factor structure of random forms and their ludged complexity. Perception \& Psychophysics, 1966, 1, 303-310.

STENSON, H. H.. KNOLL, R. L. Goodness of fit for random rankings in Kruskal's nonmetric scaling procedure. Psychological Bulletin, 1969, 71, $122-126$.

TORGERSON, W. S. Theory and methods of scaling. New York: Wiley, 1958.

TUCKER. L. R. Determination of generalized learning curves by factor analysis. ETS Technical Report, 1960. Educational Testing Service, Princeton. New Jersey.

TUCKER, L. R. Cluster analysis and the search for structure underlying individual differences in psychological phenomena. Paper presented at the Conterence on Cluster Analysis of Multivariate Data. December 1966, New Orleans, Louisiana. TUCKER, L. R, \& MESSICK, S. An individual differences model for multidimensional scaling. Psychometrika. $1963,28,333-367$.

WIGGINS, N. S., \& PRADHAN, P. L. Multidimensional scaling for individual viewpoints: A progress report. ORI Technical Report, Vol. 3, No. 3, May 1963, Oregon Research Institute.

\section{NOTE}

1. These stress values were compared with those reported by Stenson \& Knoll (1969) for 20 points in five dimensions. Stenson and Knoll obtained stress values for configurations derived from randomly generated data. Three of the above stress values are clearly acceptable by Stenson and Knoll's conservative criterion, but the other three (IIs 2, 3, and 4) are not. Several considerations influenced the decision to override the Stenson-Knoll criterion: (1) For these IIs the curves relating dimentionality to stress were well below the random curves up to some point, then the curves intersected. Does it make sense to say that a four-dimensional solution is clearly nonrandom but that a five-dimensional solution is random? (2) Data from the marker Ss for IIs 2, 3, and 4 clearly indicated a five-dimensional solution by criteria both of Krustsal and of Stenson and Knoll. (3) The relative interpretability of the results in terms of the physical measures was greatest in the five-dimensional spaces. Reducing the dimensionality of the configuration combined dimensions which were separated in the five-dimensional space. (4) Previous empirical work (the studies by Brown and by Silver. Landis, and Messick) sugfested that higher dimensional solutions would be appropriate for stimuli of this complexity.

(Accepted for publication September 22 1971.) 